\title{
Utilizing Designed Receptors Exclusively Activated by Designer Drug Chemogenetic Tools to Identify Beneficial G Protein-Coupled Receptor Signaling for Fibrosis
}

\author{
Ji Zhang, ${ }^{1}$ Eyal Vardy, ${ }^{1}$ Eric S. Muise, Tzu-Ming Wang, Richard Visconti, Ashita Vadlamudi, \\ Shirly Pinto, and Andrea M. Peier \\ Departments of Cardiometabolic Diseases (J.Z., S.P.), Screening and Compound Profiling (E.V., R.V., A.V., A.M.P.), GpGx \\ (E.S.M.), and Translational Biomarkers (T.-M.W.), MRL, Merck \& Co., Inc., Kenilworth, New Jersey; and Kallyope Inc., New York, \\ New York (E.V., S.P.)
}

Received May 12, 2020; accepted August 4, 2020

\begin{abstract}
Fibrosis or accumulation of extracellular matrix is an evolutionarily conserved mechanism adopted by an organism as a response to chronic injury. Excessive fibrosis, however, leads to disruption of organ homeostasis and is a common feature of many chronic diseases. G protein-coupled receptors (GPCRs) are important cell signaling mediators and represent molecular targets for many Food and Drug Administration-approved drugs. To identify new targets for fibrosis, we used a synthetic GPCR system named designed receptors exclusively activated by designer drugs (DREADDs) to probe signaling pathways essential for fibrotic response. We found that upon expression in human lung fibroblasts, activation of $\mathrm{Gq}$ - and Gs-DREADDs abrogated the induction of TGF $\beta$-induced fibrosis marker genes. Genome-wide transcriptome analysis identified dysregulation of multiple GPCRs in lung fibroblasts treated with TGF $\beta$. To investigate endogenous GPCR modulating TGF $\beta$ signaling, we selected 13 GPCRs that signal through Gq or Gs and activated them by using specific agonists. We examined the impact of
\end{abstract}

each agonist and how activation of endogenous GPCR affects TGF $\beta$ signaling. Among the agonists examined, prostaglandin receptor agonists demonstrated the strongest inhibitory effect on fibrosis. Together, we have demonstrated that the DREADDs system is a valuable tool to identify beneficial GPCR signaling for fibrosis. This study in fibroblasts has served as a proof of concept and allowed us to further develop in vivo models for fibrosis GPCR discovery.

\section{SIGNIFICANCE STATEMENT}

Fibrosis is the hallmark of many end-stage cardiometabolic diseases, and there is an unmet medical need to discover new antifibrotic therapies, reduce disease progression, and bring clinically meaningful efficacy to patients. Our work utilizes designed receptors exclusively activated by designer drug chemogenetic tools to identify beneficial GPCR signaling for fibrosis, providing new insights into GPCR drug discovery.

\section{Introduction}

Fibrosis is an evolutionarily conserved mechanism developed by an organism to survive chronic injury (Wynn and Ramalingam, 2012). Excessive fibrosis, however, leads to disruption of organ function and is a common feature of many chronic diseases. Many progressive forms of cardiometabolic diseases, such as end-stage renal disease, nonalcoholic steatohepatitis (NASH), and heart failure, are characterized by

\footnotetext{
This work did not receive any outside funding support.

${ }^{1}$ J.Z. and E.V. contributed equally to the work

https://doi.org/10.1124/jpet.120.000103.
}

the presence of extensive fibrosis (Friedman et al., 2013). A deeper understanding of the complex biology underlying these disease associations would greatly help the development of newer transformative therapies. Despite the broad clinical impact, there are limited treatment options for fibrosis. In 2014, the FDA approved the use of pirfenidone and nintedanib in patients with idiopathic pulmonary fibrosis (IPF), which has kindled interests in developing more antifibrotic drugs for patients. To date, several agents have demonstrated activities in reducing fibrosis in patients, including obeticholic acid, cenicriviroc, NGM282, liraglutide, and pamrevlumab (FG-3019) (Neuschwander-Tetri et al., 2015; Armstrong et al., 2016; Ratziu et al., 2016; Friedman et al., 2018; Harrison et al., 2018).

\footnotetext{
ABBREVIATIONS: BDKRB, bradykinin receptor B; BDL, bile duct ligation; CNO, clozapine N-oxide; Col1A1, collagen 1A1; DRD1, dopamine receptor D1; DREADD, designed receptors exclusively activated by designer drug; EP, E-prostanoid receptor; ESRD, end-stage renal disease; F2RL1, F2R Like Trypsin Receptor 1; FDA, Food and Drug Administration; GPCR, G protein-coupled receptor; hM3, human M3 muscarinic receptor; hM4, human M4 muscarinic receptor; GPER1, G protein-coupled estrogen receptor 1; 5-HT, 5-hydroxytryptamine; HTR, 5-HT receptor; IPF, idiopathic pulmonary fibrosis; MC1R, melanocortin receptor; NASH, nonalcoholic steatohepatitis; NHLF, normal human lung fibroblast; OXTR, oxytocin receptor; PAl-1, plasminogen activator inhibitor-1; PAR2, protease-activated receptor 2; PCR, polymerase chain reaction; PGE2, prostaglandin E2; PKA, protein kinase A; RNA -seq, RNA sequencing; PTGER4, Prostaglandin E2 receptor EP4; RT-qPCR, quantitative reversetranscription polymerase chain reaction; $\alpha \mathrm{SMA}, \alpha$-smooth muscle actin; S1PR3, sphingosine-1-phosphate receptor 3; TBXA2R, thromboxane A2 receptor; TGF $\beta$, transforming growth factor $\beta$; UUO, unilateral ureter obstruction.
} 
However, no new drug has yet been approved to stop fibrosis progression, relieve symptoms, or substantially improve patient survival. There is a huge unmet medical need to develop new antifibrosis therapies with clinically meaningful efficacy in patients.

GPCRs are important cell signaling molecules that respond to a variety of extracellular stimuli, including hormones, neurotransmitters, growth factors, chemokines, light, odor, taste, extracellular matrix, shear stress, etc. (Gilman, 1987; Hauser et al., 2017; Weis and Kobilka, 2018). GPCRs regulate nearly all physiologic activities, such as blood pressure control, respiration, hormonal-neural regulation, and immune response (Premont and Gainetdinov, 2007). It has been estimated that GPCRs comprise approximately $34 \%$ of the molecular targets for FDA-approved drugs, and $~ 60$ agents against novel GPCR targets are in clinical trials (Hauser et al., 2017). Several GPCR targeted therapies have been evaluated for fibrotic indications. Lysophosphatidic acid receptor 1 has been found linking pulmonary fibrosis to lung injury (Tager et al., 2008). A lysophosphatidic acid receptor 1 antagonist BMS-986020 was recently examined in patients with IPF (NCT01766817) and found effective at slowing down the decline of lung function (Palmer et al., 2018). Drug-related liver toxicity precluded BMS-986020 from further clinical development. Cannabinoid receptor 1 antagonism has also attracted a considerable amount of interests for the treatment of NASH because of its role in central orexigenic effect, energy expenditure, and fibrosis (Mallat et al., 2013; Patsenker and Stickel, 2016). Neuronal adverse effects of the known cannabinoid receptor 1 inverse agonist rimonabant have prompted the development of a newer peripherally restricted agent (Sam et al., 2011). Nonetheless, the interest to develop new GPCR therapies with better safety profiles for fibrosis is clearly warranted.

The activation of GPCRs under different conditions can have different biologic outcomes depending on the cellular context and conditions (Gilman, 1987; Weis and Kobilka, 2018). Identification of beneficial GPCR signaling pathways for fibrosis will enable more focused target identification and drug screening efforts. Upon activation, a GPCR binds to a heterotrimeric G protein. The $\alpha$-subunit of the G protein is subsequently dissociated from the $\beta \gamma$-subunits to mediate downstream signals (Weis and Kobilka, 2018). There are four main subtypes of $\alpha$-subunit: Gq/11, Gs, Gi/o, and G12/13. Gq/ 11 protein couples GPCRs to phospholipase C- $\beta$, which in turn hydrolyzes phosphatidylinositol 4,5-bisphosphate to second messengers diacyl glycerol and inositol trisphosphate. Inositol trisphosphate stimulates calcium release into the cytoplasm. Gs protein binds to adenylyl cyclase to produce cAMP and activate protein kinase A (PKA) signaling. On the other hand, Gi/o protein primarily inhibits PKA signaling by inhibiting adenylyl cyclase activity and decreasing cAMP production. G12/13 protein regulates actin cytoskeletal remodeling in cells.

The designed receptors exclusively activated by designer drugs (DREADDs) system has been recently developed to delineate $\mathrm{G}$ protein signaling underlying biologic responses (Urban and Roth, 2015). DREADDs are synthetic GPCRs that have been engineered to respond to otherwise inert ligands, such as clozapine N-oxide (CNO) (Urban and Roth, 2015). Different DREADDs have been engineered to selectively activate Gq, Gs, or Gi pathways. By using the DREADD chemogenetic tools, we found that $\mathrm{Gq}$ and Gs signaling decreases cellular response to TGF $\beta$. Whole-genome transcriptome analysis of human primary fibroblasts and fibrosis models revealed 13 highly dysregulated GPCRs that signal through either Gq or Gs pathways with known agonists. We further showed that among those GPCRs, activation of the prostaglandin receptors reduced fibrotic marker expression in fibroblasts. The combination of DREADD chemogenetic tools with transcriptome analysis represents a powerful tool for identifying potential GPCR targets for fibrosis.

\section{Materials and Methods}

Cultured Cells and Reagents. Normal human lung fibroblasts (CC-2512, lot 0000343490; Lonza) were cultured in fibroblast basal medium (CC-3131; Lonza) with growth supplements (CC-4126; Lonza). Human primary cardiac fibroblasts (6300, lot 5433; Sciencell) were cultured in fibroblast medium 2 (FM2) media (2331; Sciencell) supplemented with FBS (0025; Sciencell), Fibroblast Growth Supplement 2 (FGS-2) (2382; Sciencell), and penicillin-streptomycin (15070063; Life Technologies). Human primary renal proximal tubule epithelial cells (CC-2553, lot 0000362300; Lonza) were cultured in renal epithelial cell growth basal medium (CC-3191; Lonza) supplemented with REGM SingleQuots (CC-4127; Lonza). Primary human hepatic stellate cells (5300, lot 10279; Sciencell) were cultured in Stellate Cell Medium (5301; Sciencell) supplemented with FBS (0010; Sciencell), stellate cell growth supplement (5352; Sciencell), and penicillin-streptomycin solution (0503; Sciencell). All cells were incubated at $37^{\circ} \mathrm{C}$ in the presence of $5 \% \mathrm{CO}_{2}$.

hM3-Gq-DREADD, hM4-Gi-DREADD, hM3-Gs-DREADD, and kappa opioid receptor or KOR-Gi-DREADD were cloned into a BacMam vector. The viruses were expressed and purified by Life Technologies. For TGF $\beta$ treatment, cells were plated and serumstarved overnight. The next day, $5 \mathrm{ng} / \mathrm{ml}$ of recombinant human TGF $\beta 1$ (580702; BioLegends) was added to the culture medium, and cells were incubated at $37^{\circ} \mathrm{C}$ for the indicated time. For activation of DREADDs, cells were infected with control or DREADD-expressing BacMam viruses at various titers for 24 hours. Cells were subsequently treated with $\mathrm{CNO}$ or salvinorin $\mathrm{B}$ in the presence of recombinant human TGF $\beta 1$ for 24 hours. SB-525334, CNO, salvinorin B, bradykinin, prostaglandin E2 (PGE2), oxytocin, and endothelin 3 were from Sigma. G-1, A68930, TCS2510, U46619, WAY267464, AC264613, CYM5541, BW723C86, AS19, and BMS470539 were from Tocris Biosciences. For inhibitor treatment, cells were treated with recombinant human TGF $\beta 1$ in the presence of vehicle DMSO (D2650; Sigma) or compounds at various concentrations.

cAMP and Calcium Signaling. At 24 hours after BacMam viral infection, cells were treated with $\mathrm{CNO}$ at various concentration for 30 minutes. After removing media, cAMP stimulation or inhibition of basal levels of cAMP were measured by cAMP Gs Dynamic kit (62 AM4PEB; Cisbio) or Gi kit (62 AM9PEB; Cisbio). For calcium measurement, cells were incubated with fluorescence dye from FLIPR Calcium 5 Assay Kit (R8185; Molecular Devices) for 1 hour at $37^{\circ} \mathrm{C}$. The plate was then set up in the FLIPR Tetra highthroughput cellular screen system, treated with CNO, and read immediately.

Rodent Fibrosis Models. Adult male C57BL/6 mice (Jackson Laboratory, Bar Harbor, ME) and adult male Sprague-Dawley rats (Taconic, Rensselaer, NY) were housed in a temperature- and humidity-controlled facility with a 12-hour light/dark cycle. Animals had ad libitum access to food (Purina Rodent Chow 5053; LabDiet, St. Louis, MO) and water. All procedures utilizing experimental animals were conducted in accordance with the Guide for the Care and Use of Laboratory Animals, and experimental procedures were reviewed 
and approved by the Institutional Animal Care and Use Committee at MRL, Kenilworth, NJ. Mouse UUO kidney fibrosis and carbon tetrachloride-induced liver fibrosis models as well as rat bile duct ligation models were carried out as previously described (Zhang et al., 2020).

Gene Expression Taqman Analysis. Total RNA was extracted from cultured cells or tissues following the manufacturer's instructions for RNeasy Mini QIAcube Kit (74116; Qiagen). Two micrograms of RNA was then reverse-transcribed using SuperScript VILO cDNA Synthesis kit (11754-050; Invitrogen). Two microliters of diluted cDNA was added to quantitative real-time PCR mix with TaqMan Universal PCR Master mix (4364338; Applied Biosystems) and Taqman probes (Life Technologies). PCR amplification (one cycle at $50^{\circ} \mathrm{C}$ for 2 minutes, one cycle at $95^{\circ} \mathrm{C}$ for 10 minutes, and 40 cycles at $95^{\circ} \mathrm{C}$ for 15 seconds and $60^{\circ} \mathrm{C}$ for 1 minute) was done in an Applied Biosystems 7900HT Fast Real-Time PCR System (Applied Biosystem). The cycle threshold or $\mathrm{Ct}$ value of each sample was normalized to the glyceraldehyde 3-phosphate dehydrogenase $($ GAPDH ) level and quantified based on $2 \mathrm{e}-\Delta(\Delta \mathrm{Ct})$ method (comparing with no-treatment or sham group).

RNA Isolation and Gene Expression Profiling. Gene expression analysis was performed as previously described (Schlessinger et al., 2015). Briefly, sequencing was performed using the Truseq stranded total RNA RiboZero library preparation kit (RS-122-2201; Illumina, San Diego, CA) according to the manufacturer's instructions (Illumina). The resulting cDNA libraries were sequenced on an Illumina (HiSeqTM 4000) using a 50-base paired-end run. Alignment and differential gene expression analysis was performed in Omicsoft Array Studio version 9.0.8.92. Briefly, cleaned reads were aligned to the mouse and human B38 genome references for the UUO kidney and primary cells. respectively, using the Omicsoft Aligner with a maximum of two allowed mismatches. Gene level counts and Fragments Per Kilobase (FPKM) were determined by the Omicsoft sequence aligner (OSA) algorithm as implemented in Omicsoft Array Studio and using Ensembl.R82 gene models. At least 90\% of reads across all samples mapped to the respective genome (corresponding to 55-80 million reads). Differential gene expression analysis was performed by the DESeq2 algorithm as implemented in Omicsoft Array Studio, with the samples from the shamoperated or vehicle-treated animals serving as reference, yielding fold change and corrected $P$ values (false discovery rate, Benjamini-Hochberg). Gene Ontology (GO) term enrichment analysis was performed using the PANTHER enrichment test (http:// pantherdb.org). Heatmaps and box plots were created using Omicsoft Array Studio.

Data Analysis. All data are presented as means \pm S.E.M. One- or two-way ANOVA post hoc Tukey analysis was used for data comparisons, as indicated in the figure legends, together with group size. All analyses were done using Prism 7 software. A $P$ value of $<0.05$ was statistically significant.

\section{Results}

Establishment of Functional DREADDs Expression System in Primary Human Lung Fibroblasts. DREADDs are synthetic GPCRs whose expression does not affect the biologic system when not activated by their highly specific synthetic ligands. Gq-DREADD (hM3Dq) was created by directed molecular evolution of the human M3 muscarinic receptor (hM3) in yeast (Armbruster et al., 2007). When activated by the ligand $\mathrm{CNO}$, it stimulated calcium release into the cytoplasm. Gi-DREADD (hM4Di) was designed based on the human M4 muscarinic receptor; it decreased cAMP signaling and opens $\mathrm{K}^{+}$channels ( $\mathrm{G}$ protein-coupled inwardly rectifying potassium channels) in response to CNO (Nawaratne et al., 2008). A chimeric hM3Dq $\beta$-adrenergic receptor DREADD was created as Gs-DREADD, which activated cAMP production and PKA signaling (Guettier et al., 2009). Unlike hM3Dq and hM4Di, Gs-DREADD has a modest degree of constitutive activity, which can lead to basal phenotypes in certain cellular contexts.

BacMam is a highly effective viral expression system that enables protein expression in a large variety of cells, including human primary cells (Ames et al., 2007). Gq/Gs/Gi-DREADDs were cloned into a bi-cistronic BacMam vector that simultaneously expresses mCitrine fluorescent protein under the control of internal ribosome entry site (Fig. 1A). At 24 hours after BacMam infection, the expression of DREADDs was confirmed by the coexpressed fluorescent signals in normal human lung fibroblasts (NHLFs) (Fig. 1B). At the optimal viral titers, about $50 \%$ of cells were positive for fluorescence. To verify the function of exogenously expressed DREADDs, we next examined calcium signaling in all cells. As shown in the top panel of Fig. 1C, CNO treatment induced a dosedependent spike of calcium release into the cytoplasm of Gq-DREADD-expressing fibroblasts. cAMP production was induced by CNO in Gs-DREADD-expressing fibroblasts (Fig. 1C, middle panel). Conversely, activation of Gi-DREADD-expressing fibroblasts by CNO led to an expected, modest reduction of the basal cAMP level (Fig. 1C, bottom panel). These results indicated that the BacMam-delivered DREADD expression system was functional in those cells.

Activation of Gq and Gs Signaling Inhibits the Expression TGF $\beta$-Induced Fibrosis Marker Genes. Among many factors that regulate fibrosis, TGF $\beta$ plays an essential role in both myofibroblast activation and extracellular matrix remodeling (Akhurst and Hata, 2012); it has often been used to induce fibrotic response in cultured cells. To gain novel insight into the GPCR pathways modulating TGF $\beta$, we next established a cell-based assay to examine $\mathrm{TGF} \beta$ response (Fig. 2A). At 24 hours after TGF $\beta$ treatment, RT-qPCR analysis of fibrotic marker genes, such as $\alpha$-smooth muscle actin ( $\alpha$ SMA), PAI-1, and collagen 1A1, in NHLFs revealed a robust and statistically significant response to $\mathrm{TGF} \beta$, which was almost completely abrogated in the presence of SB525334 , a TGF $\beta$ type I receptor (activin receptor-like kinase 5) inhibitor (Fig. 2A).

To test the effect of DREADD activation on fibrosis markers in those cells, we infected the NHLFs with DREADDexpressing BacMam viruses and treated NHLFs with various concentration of $\mathrm{CNO}$ in the presence of TGF $\beta$. After a treatment period of 24 hours, the cells were collected for mRNA extraction and RT-qPCR analysis. TGF $\beta$-induced transcription of $\alpha$ SMA, PAI-1, and collagen 1A1 (Col1A1) was suppressed by activation of Gq- and Gs-DREADDs. CNO had no effect on these markers in NHLFs infected with Gi-DREADD. A clear dose-dependent CNO effect was observed for all markers in Gs-DREADD-expressing NHLFs, whereas for Gq-DREADD-expressing cells, a clear dose-dependent effect was only observed for $\alpha$ SMA, only a minimal effect on Col1A1, and a saturated significant effect on PAI-1 (Fig. 2B).

RNA-Seq Analysis Identifies Dysregulated GPCRs in NHLFs upon TGF $\beta$ Treatment. Notably, many GPCRs function through Gq and Gs signaling, such as 5-HT2 receptors and prostaglandin receptors (Fribourg et al., 2011). To identify endogenous GPCRs that modulate TGF $\beta$ signaling, we resorted to our previous genome-wide transcriptome 
A

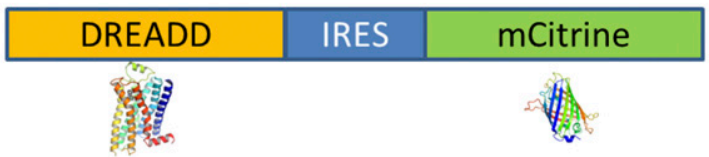

B
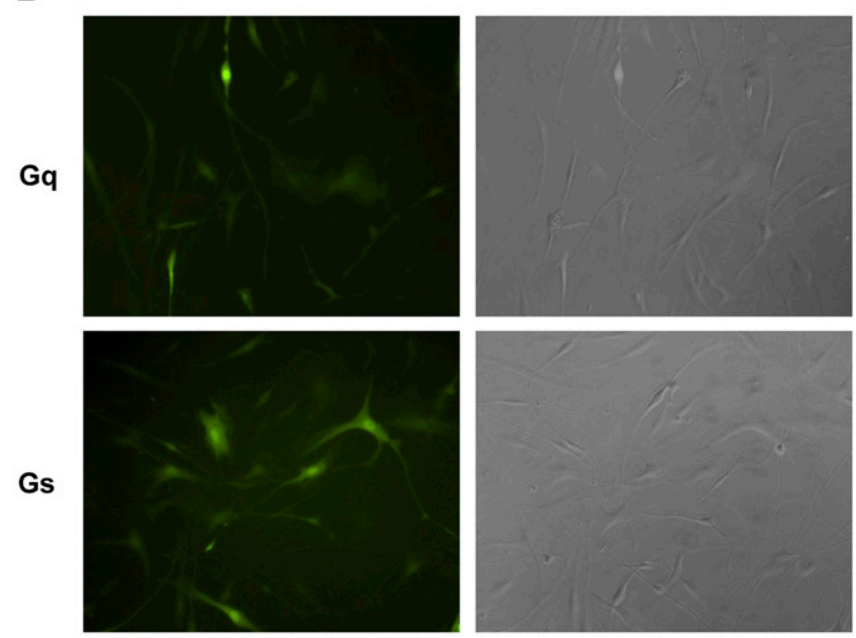

Gi

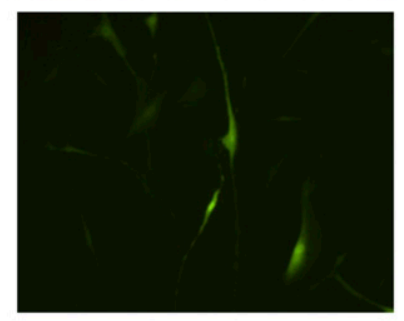

C

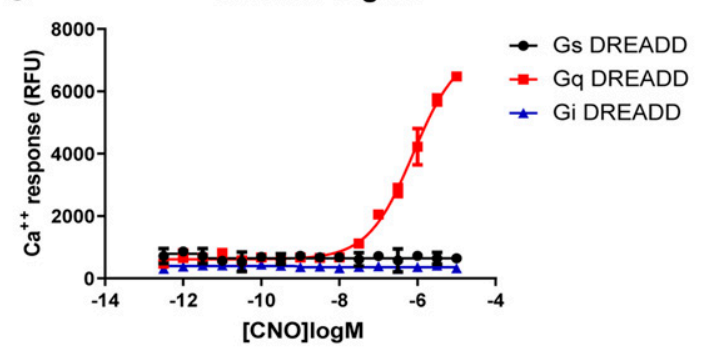

cAMP Signal

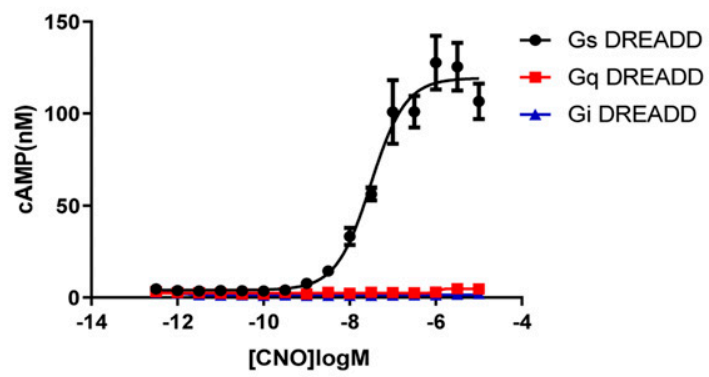

cAMP Signal

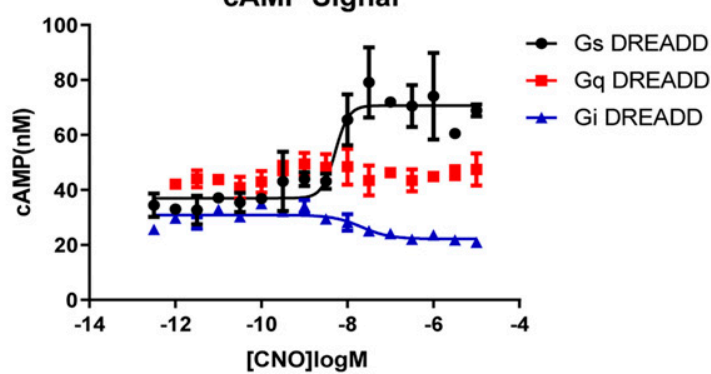

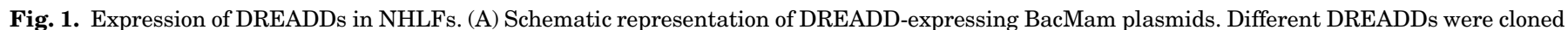

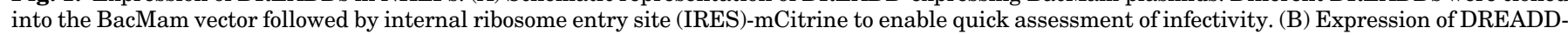

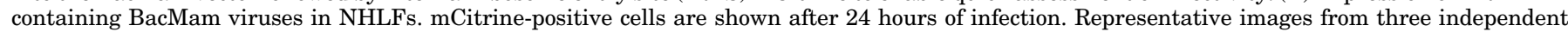

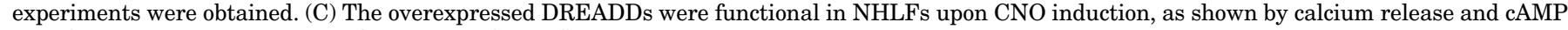
signaling assays. $N=3$, mean \pm S.D. RFU, relative fluorescence unit.

analysis of human primary cells treated with TGF $\beta$ to uncover differentially expressed GPCRs under TGF $\beta$ stimulation (manuscript accepted at Cell Reports Medicine, National Center for Biotechnology Information (NCBI) Gene Expression Omnibus (GEO) accession number GSE152250) (Zhang et al., 2020). Upon TGF $\beta$ treatment, human primary cardiac fibroblasts, NHLFs, hepatic stellate cells, and renal proximal tubular epithelial cells were collected for RNA-seq analysis. Among the four cell types, the expression of many nonsensory GPCR genes was significantly changed upon TGF $\beta$ treatment when compared with the vehicle group (Fig. 3A). To delineate this further, a volcano plot visualization of GPCR expression fold change in NHLFs is shown in Fig. 3B. We highlighted 13 GPCRs that signal through Gq or Gs pathways and have known biologic functions relevant to fibrosis and commercially available agonists (Table 1). Among the selected GPCRs, S1PR3, HTR2B, BDKRB1, BDKRB2, TBXA2R, PTGER2, and GPER1 were significantly decreased upon TGF $\beta$ treatment in lung fibroblasts, whereas DRD1, PAR2 (F2RL1), and PTGER4 were significantly upregulated (Fig. 3B). Furthermore, we examined the expression of this set of GPCRs in unilateral ureter obstruction kidney, carbon tetrachloride liver, and bile duct ligation (BDL) liver fibrosis models. As shown in the heatmap of Fig. 3C, the expression of many GPCRs was reduced in primary cells treated with TGF $\beta$ but augmented in fibrotic tissues, suggesting additional layers of complexity for the regulation of GPCR.

Reversal of Fibrosis by Prostaglandin Receptors in NHLFs. We have found a subset of Gq- or Gs-coupled GPCRs whose expression was differentially regulated in lung fibroblasts treated with TGF $\beta$. We next sought to examine whether activation of those endogenous GPCRs would alter TGF $\beta$ signaling. To this end, we obtained 14 potent and selective agonists for those GPCRs with reported $\mathrm{EC}_{50}$ at a low nanomolar range (Table 1). NHLFs were first treated with CNO at various concentrations. Calcium signal and cAMP production were determined to ensure selective $G$ protein pathway activation. As shown in Fig. 4A, dose-dependent calcium release was detected in cells treated with bradykinin and oxytocin at a statistically significant level. These results suggested that BDKRB1/2 and OXTR are the major Gq-coupled endogenous receptors in NHLFs. Moreover, cAMP production was substantially elevated in cells treated with A68930 (DRD1 agonist), 
A

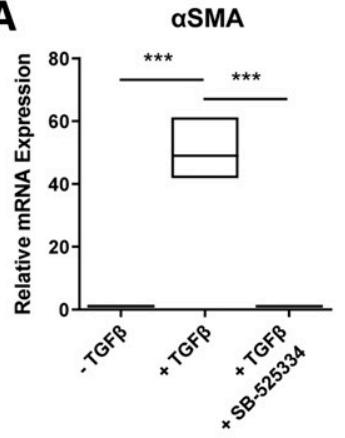

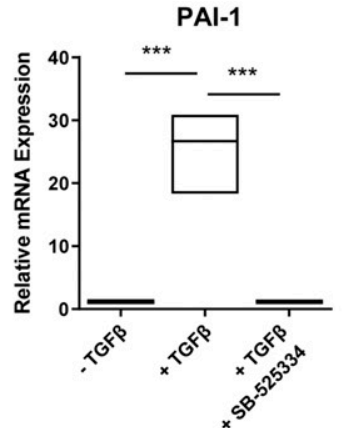

Col1A1

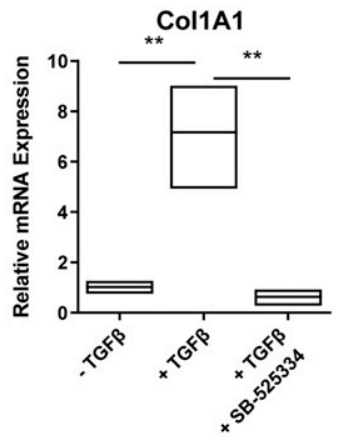

B

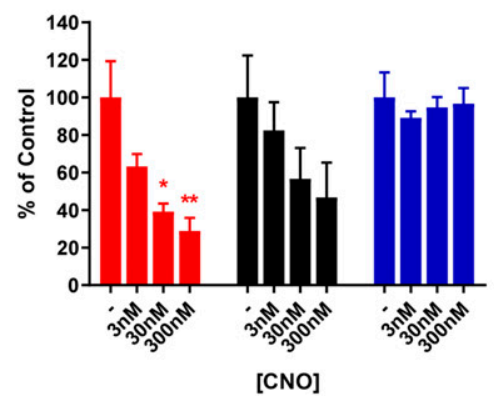

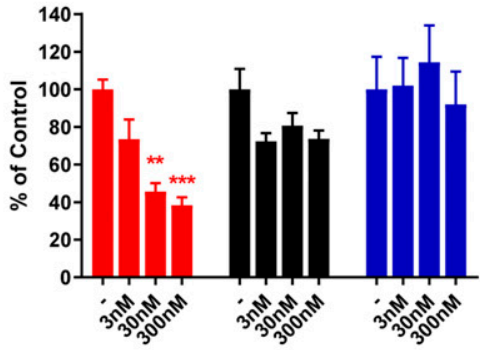

[CNO]

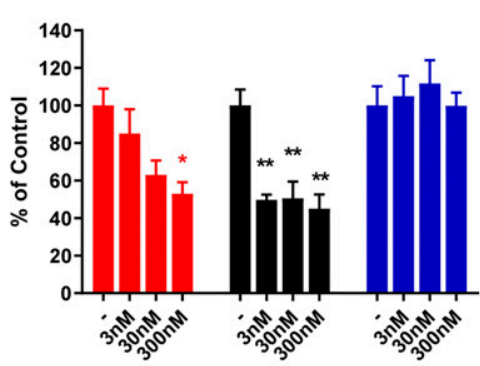

[CNO]

Fig. 2. Activation of $\mathrm{Gq}$ and Gs signaling inhibits TGF $\beta$-induced fibrosis marker genes in lung fibroblasts. (A) $\alpha$ SMA, Col1A1, and PAI-1 genes were markedly upregulated after TGF $\beta$ treatment $(5 \mathrm{ng} / \mathrm{ml}$ for 24 hours $)$ in NHLFs, which was almost completely reversed by the coadministration of activin receptor-like kinase 5 inhibitor SB-525334 $(10 \mu \mathrm{M}) . N=3$, mean \pm S.D. $* P<0.05 ; * * P<0.005 ; * * * P<0.001$, compared with no CNO treatment. Oneway ANOVA post hoc Tukey test. Three independent replicates were obtained. (B) Activation of Gq and, to a lesser extent, Gs by CNO inhibits $\alpha$ SMA, Col1A1, and PAI-1 expression in NHLFs. $N=3$, mean \pm S.D. $* P<0.05 ; * * P<0.005$; $* * P<0.001$, compared with no CNO treatment. One-way ANOVA post hoc Tukey test. Three independent replicates were obtained.

prostaglandin E2 (EP agonist), and TCS2510 (EP4 agonist), consistent with their role in Gs-mediated signaling (Fig. 4B). U46619 treatment induced a dose-dependent increase of cAMP level, albeit with lower overall signals, indicating an alternative signaling pathway than the previously reported Gq signaling. WAY267464 (OXTR agonist), AC264613 (PAR2 agonist), CYM5541 (S1PR3 agonist), and BW723C86 (5-HT2B/C agonist) treatment led to a slight reduction of cAMP, indicative of $\mathrm{Gi}$ activation. Those observations were inconsistent with their reported Gq pathway.

Subsequently, we measured the expression of various fibrosis marker genes in those cells treated with $100 \mathrm{nM}$ or $1 \mu \mathrm{M}$ of compounds in the presence of TGF $\beta$ (Fig. 4C). As expected, TGF $\beta$ substantially induced $\alpha$ SMA gene expression (top panel, orange bar). The addition of bradykinin, A68930 (DRD1 agonist), U46619 (TBXA2R agonist), and oxytocin were not able to suppress $\alpha$ SMA expression. However, treating cells with prostaglandin/PGE2 and TCS2510 (EP4 agonist) dramatically reduced $\alpha$ SMA level at both concentrations. Endothelin-3 treatment inhibited $\alpha$ SMA expression at the lower concentration $(100 \mathrm{nM})$ but not the higher concentration $(1 \mu \mathrm{M})$. The impact of agonist treatment on TGF $\beta$-induced Col1A1 gene expression was also examined. PGE2, TCS2510 (EP4 agonist), and oxytocin led to a dose-dependent inhibition of Col1a1 expression (Fig. 4C, middle panel). Among which, PGE2-mediated inhibition of Col1A1 is more substantial. PAI-1 is another important TGF $\beta$ target gene. RT-qPCR analysis revealed that PGE2 and oxytocin potently suppressed PAI-1 induction upon TGF $\beta$ treatment (Fig. 4C, bottom panel). Overall, our results found that the prostaglandin agonist activates Gs signaling and has the strongest antifibrotic effect in NHLFs.

\section{Discussion}

In the present study, we used DREADD chemogenetic tools to probe GPCR signaling pathway(s) essential for fibrotic response in fibroblasts. We discovered that activation of either Gq or Gs pathways inhibits TGF $\beta$-induced fibrosis marker gene expression. Stimulation of the Gi-DREADD did not affect transcription of these marker genes. However, the current experiments cannot completely rule out the possibility that a low level of hM4D expression is the reason we could not see a response. The reduction of basal levels of cAMP we measured, although statistically significant, was small and may suggest a low level of expression.

To identify endogenous Gq/Gs-coupled GPCR in fibroblasts, we performed datamining of our fibrosis profiling dataset, in which whole-genome transcriptome analyses were carried out in human primary cardiac fibroblasts, NHLFs, hepatic stellate cells, and renal proximal tubular epithelial cells treated with TGF $\beta$, as well as UUO kidney, carbon tetrachloride liver, and BDL liver fibrosis models (Zhang et al., 2020). Among the highly differentially expressed GPCRs, we selected 13 receptors that were signaling through $\mathrm{Gq}$ or $\mathrm{Gs}$ and have a known agonist available. We further verified the expression and activation of those GPCRs by agonist stimulation in the fibroblasts. Among the 13 GPCRs, activation of prostaglandin receptors using the panagonist prostaglandin generated the strongest antifibrotic effect. Activation of only one of the prostaglandin receptors (EP4) using TCS2510 resulted in a reduced antifibrotic effect, suggesting the requirement of multiple prostaglandin receptors for optimal antifibrotic effect.

Although we refer to the signaling pathways of the DREADDs based on the $\mathrm{G} \alpha$-subunit they interact with, the signaling 
A
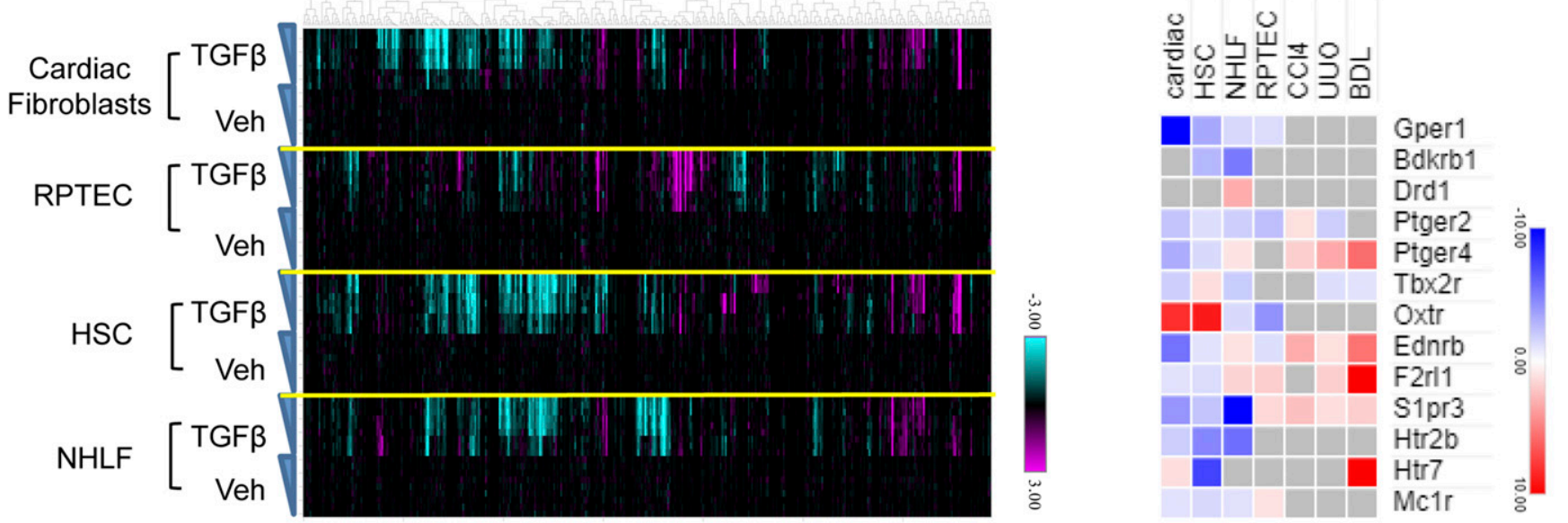

B

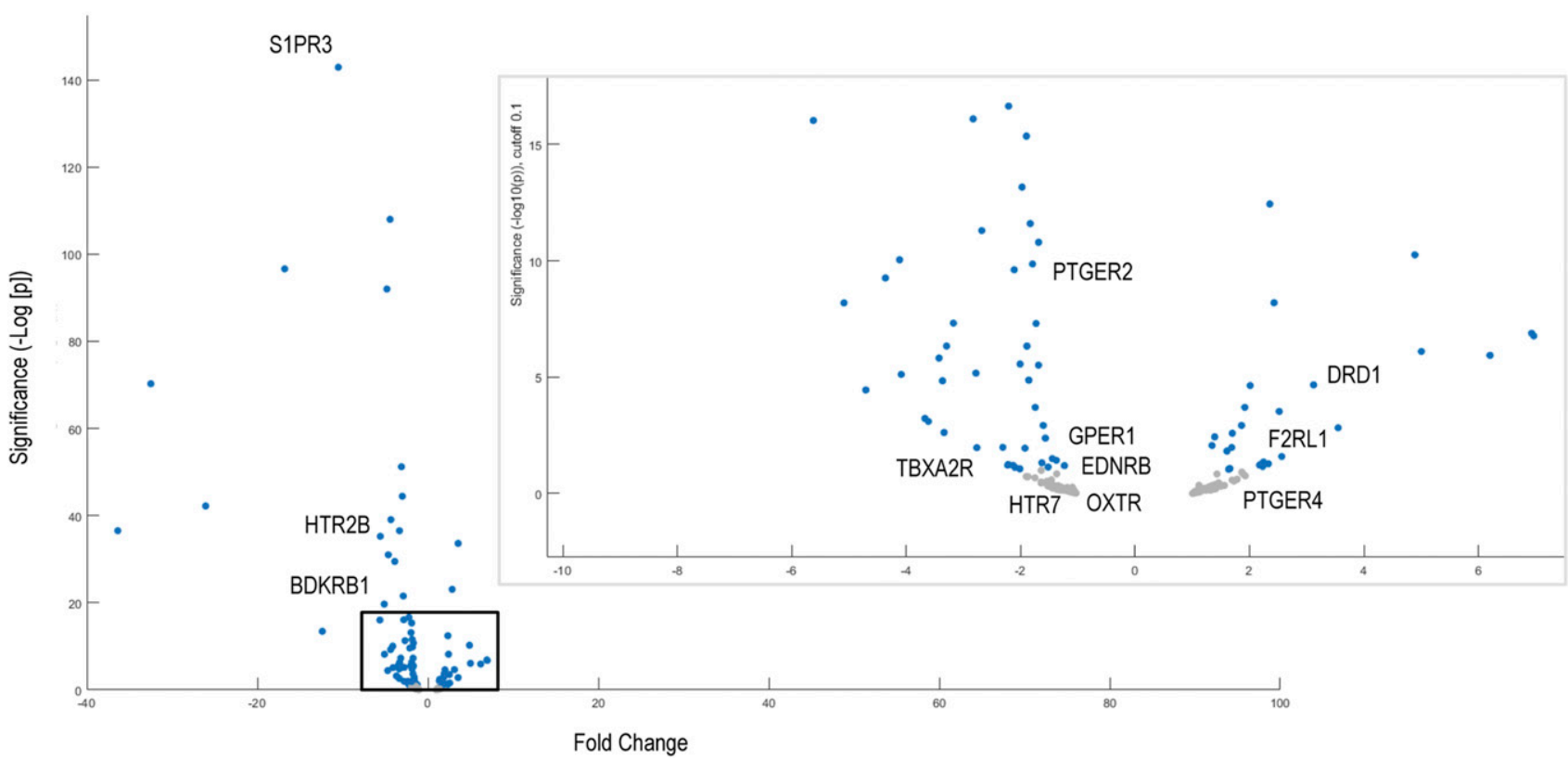

Fig. 3. RNA-seq analysis identifies dysregulated GPCRs in NHLFs upon TGF $\beta$ treatment. (A) Expression profile of nonsensory GPCRs in human primary cells treated with vehicle or with $\mathrm{TGF} \beta(5 \mathrm{ng} / \mathrm{ml})$. The color gradient represents the fold change compared with vehicletreated cells (-3.0- to 3.0-fold). $N=3$ for each time point or treatment group. (B) Volcano plot of GPCR expression in NHLFs. (C) Gene expression ratios of selected GPCRs in human primary cells and fibrotic tissues. From left: NHLFs, cardiac fibroblasts, hepatic stellate cells (HSCs), renal proximal tubular epithelial cell (RPTEC), UUO kidney, CCl4 liver, and BDL liver. Heatmap was generated by using Morpheus software (https://software.broadinstitute.org/morpheus/). The color gradient represents fold change comparing treatment (TGF $\beta 24$ hours, surgery, or carbon tetrachloride treatment) with control groups (-10- to 10-fold). Gray indicates below detection limit. EDNRB, endothelin receptor B; Veh, vehicle.

pathways activated by them are likely more complicated and include components of $\beta \gamma$ signaling, arrestin signaling, and potentially other downstream molecules that may affect transcription factors in a cumulative manner. The definition of receptors as coupled to Gq or Gs does not necessarily mean that this is the only $\mathrm{G} \alpha$ protein they interact with, but it is the most common one. In many cases, these definitions rely on the cellular effects seen by activation of a specific receptor based on cAMP or $\mathrm{Ca}^{2+}$ response. The endogenous receptors may have different effects on some of the signaling pathways because of different coupling preferences or alternative signaling pathways that were not monitored in the current experiment.
Signal intensity in cellular system is another factor that needs to be considered when discussing downstream physiologic effects. It is important to understand that in an artificial system overexpressing a GPCR, the signal intensity is often higher than what is generated by activation of GPCRs naturally expressed in those cells due to stochiometric considerations of the signal producing process. Both the intensity and subcellular location of the signal may play a role in the type and amount of physiologic response. Even when considering the limitation of the DREADD system, our results have demonstrated that it is a valuable tool to investigate beneficial GPCR signaling for fibrosis and other physiologic phenomena. 
TABLE 1

Selected GPCRs that function through Gq and/or Gs pathways

Their agonists were reported with $\mathrm{EC}_{50}$ value.

\begin{tabular}{|c|c|c|c|c|}
\hline GPCR & Signaling & Functions & Agonists & $\mathrm{EC}_{50}$ or $\mathrm{Kd}$ \\
\hline GPER1 (Gpr30) & $\mathrm{Gq}$ & $\begin{array}{c}\text { Activated by estradiol and functions via Gq signaling } \\
\text { (Revankar et al., 2005). A selective agonist G1 is beneficial } \\
\text { in myocardial ischemia reperfusion injury model (Bologa } \\
\text { et al., 2006; Deschamps and Murphy, 2009). }\end{array}$ & G1 & $2 \mathrm{nM}$ (Bologa et al., 2006) \\
\hline BDKRB1 & $\mathrm{Gq}$ & $\begin{array}{l}\text { BDKRB1 is a Gq-coupled GPCR, whereas BDKRB2 signals } \\
\text { through Gq and Gi. In vivo activation of BDKRB2 } \\
\text { attenuated kidney fibrosis (Schanstra et al., 2002). }\end{array}$ & Bradykinin & $3.26 \mathrm{nM}$ (Wiernas et al., 1997) \\
\hline DRD1 & Gs & $\begin{array}{l}\text { Pleuropulmonary fibrosis was linked to the long-term use } \\
\text { of dopamine agonist pergolide in patients with Parkinson } \\
\text { (Tintner et al., 2005). }\end{array}$ & A68930 & $2.5 \mathrm{nM}$ (DeNinno et al., 1991) \\
\hline PTGER2 & $\mathrm{Gq}$ & $\begin{array}{l}\text { PGE2 is a potent bioactive eicosanoid that strongly inhibits } \\
\text { fibroblast proliferation, migration, and collagen secretion } \\
\text { (Bozyk and Moore, 2011). }\end{array}$ & PGE2 & $\begin{array}{l}\text { PTGER1, } 25 \mathrm{nM} \text {; PTGER2, } 13 \mathrm{nM} \\
\text { PTGER3, } 3 \mathrm{nM} \text {; PTGER4, } 3 \mathrm{nM} \\
\text { (Markovič et al., 2017) }\end{array}$ \\
\hline PTGER4 & Gs & TCS2510 is a highly selective EP4 agonist. & TCS2510 & $2.5 \mathrm{nM}$ (Billot et al., 2003) \\
\hline TBXA2R & $\mathrm{Gq} / \mathrm{Gs}_{\mathrm{s}} / \mathrm{G}_{12 / 13}$ & $\mathrm{U} 46619$ is a potent and stable TBXA2R agonist. & U46619 & 35 nM (Coleman et al., 1981) \\
\hline OXTR & $\mathrm{Gq}$ & $\begin{array}{c}\text { Oxytocin is a peptide hormone and neurotransmitter } \\
\text { signaling through OXTR and Gq pathways (Gimpl and } \\
\text { Fahrenholz, 2001). WAY-267464 is a potent, selective, } \\
\text { nonpeptide OXTR agonist. }\end{array}$ & $\begin{array}{c}\text { Oxytocin } \\
\text { WAY267464 }\end{array}$ & $\begin{array}{l}\text { 9.0-10.8 nM (Gruber et al., 2012) } \\
\text { 9.0 nM (Ring et al., 2010) }\end{array}$ \\
\hline EDNRB & $\mathrm{Gq}$ & $\begin{array}{c}\text { Endothelin } 3 \text { is a vasoconstrictor that preferentially binds } \\
\text { to EDNRB. }\end{array}$ & Endothelin 3 & $1 \mathrm{nM}$ (Sumner et al., 1992) \\
\hline F2RL1 (PAR2) & $\mathrm{Gq} / \mathrm{Gi}$ & $\begin{array}{c}\text { PAR2 is implicated in lung, kidney, liver, and cardiac } \\
\text { fibrosis (Palygin et al., 2016; Shearer et al., 2016; Sun } \\
\text { et al., 2017; Friebel et al., 2019). }\end{array}$ & AC264613 & 31 nM (Gardell et al., 2008) \\
\hline S1PR3 & $\mathrm{Gq}$ & $\begin{array}{l}\text { S1PR3 KO mice has attenuated inflammation and fibrosis } \\
\text { upon bleomycin-induced lung injury (Murakami et al., } \\
\text { 2014). }\end{array}$ & CYM5541 & 72-132 nM (Jo et al., 2012) \\
\hline $\begin{array}{l}\text { HTR2B } \\
\text { (5-HT2B } \\
\text { receptor) }\end{array}$ & $\mathrm{Gq}$ & $\begin{array}{l}\text { Serotonin fibrosis in human and treatment of } \\
\text { fenfluramine, a serotonin releasing agent, causes cardiac } \\
\text { valve fibrosis in patients with obesity (Roth, 2007). 5-HT2B } \\
\text { activation mediates valvular fibroblasts activation. }\end{array}$ & BW723C86 & $\begin{array}{l}\text { 5-HT2B, } 1.1 \mathrm{nM} \text {; 5-HT2C, } 93.3 \mathrm{nM} \\
\text { (Porter et al., 1999) }\end{array}$ \\
\hline $\begin{array}{l}\text { HTR7 }(5-\mathrm{HT} 7 \\
\text { receptor) }\end{array}$ & Gs & AS-19 is a potent 5-HT7 receptor agonist. & AS19 & $\begin{array}{l}0.83 \mathrm{nM}(\text { Perez-Garcia and } \\
\text { Meneses, 2005) }\end{array}$ \\
\hline MC1R & Gs & $\begin{array}{l}\text { MC1R is anti-inflammatory and has been implicated in } \\
\text { several inflammatory diseases. }\end{array}$ & BMS470539 & $16.8 \mathrm{nM}$ (Kang et al., 2006) \\
\hline
\end{tabular}

EDNRB, endothelin receptor B; KO, knockout.

Many studies have investigated the molecular mechanisms underlying fibrosis, and a wide range of targets have been tested for drug discovery and clinical development (Wynn and Ramalingam, 2012; Friedman et al., 2013; Duffield, 2014; Nanthakumar et al., 2015). Regardless of disease etiology, fibrosis in the heart, lung, kidney, liver, and skin shares many similar features, such as increases in myofibroblast activation, collagen production, and inflammation, suggesting the existence of common core pathways/mechanisms among all fibrotic tissues. Our study focused on probing common GPCR signaling in the fibroblasts and identified Gs signaling as an important pathway for fibrosis, consistent with previously reported modulation of TGF $\beta$ signaling by cAMP (Schiller et al., 2010). DREADDs are synthetic GPCRs activated by synthetic small molecules that exhibit no biologic activity at native receptors so that they can be engineered to transduce a specific signaling in response to otherwise inert ligands in vitro and in vivo. Identification of GPCR signaling pathways that have desired outcomes in a given phenotypic assay along with transcriptional analysis of the disease state may greatly facilitate target identification and enable more focused drug discovery efforts.

About $34 \%$ of the FDA-approved drugs are targeting against GPCRs (Hauser et al., 2017). Fibrosis is associated with endstage organ damage and worsening disease progression in patients with NASH, end-stage renal disease, heart failure, and IPF. There is a huge unmet medical need to develop new fibrosis therapies that bring clinically meaningful efficacy to patients. The interest in discovering a new GPCR target for fibrosis is conceivable. Our work highlighted the importance of prostaglandin signaling in the fibroblasts. PGE2 is a locally active eicosanoid that modulates vascular tone, coagulation homeostasis, and inflammation. PGE2 binds to and activates family of GPCR receptors, E-prostanoid receptor (EP) 1, EP2, EP3, and EP4 in an autocrine or paracrine manner (Elwakeel et al., 2019). It has been shown that lung fibroblasts isolated from patients with IPF have diminished expression of PGE2 (Wilborn et al., 1995). PGE2 inhibits fibroblast proliferation and myofibroblast activation via EP2 and PKA-dependent, Smad-independent pathway (Lama et al., 2002; Kolodsick et al., 2003; Thomas et al., 2007; Wettlaufer et al., 2016). Additionally, PGE2 has been shown to work through serumresponse factor-myocardin-related transcription factor-A to regulate $\alpha$ SMA expression (Penke et al., 2014). The EP2 agonist butaprost attenuates fibrosis in UUO kidneys and human kidney slices (Jensen et al., 2019). Recent highthroughput myofibroblast phenotypic screen assay also finds EP2 and EP4 agonists beneficial for the treatment of lung fibrosis (Sieber et al., 2018). The role of PGE2 in inflammation is complex: it is proinflammatory in the early stages of inflammation but promotes the resolution of inflammation at a later time point (Calder, 2009). The 

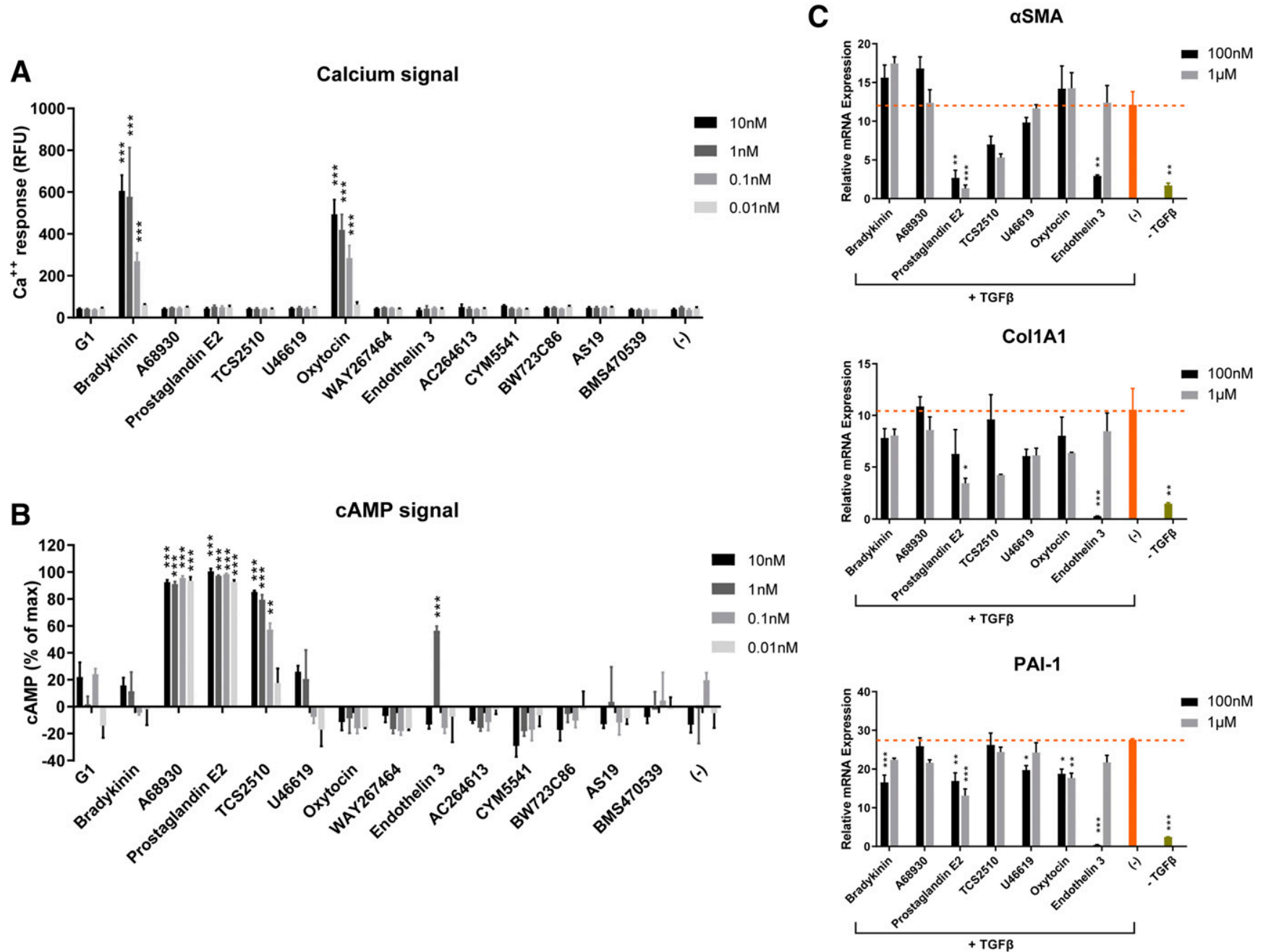

Fig. 4. Reversal of fibrosis by prostaglandin receptors in NHLFs. (A) Dose titration of various agonists to identify functional GPCRs for Gq activation in NHLFs. $N=3$, mean \pm S.D. $* P<0.05 ; * * P<0.005$; $* * * P<0.001$, compared with no compound treatment group. Two-way ANOVA post hoc Tukey test. Three independent replicates were obtained. (B) Dose titration of various agonists to identify functional GPCRs for Gs or Gi activation in NHLFs. $N=3$, mean \pm S.D. $* P<0.05 ; * * P<0.005 ; * * *<0.001$, compared with no compound treatment group. Two-way ANOVA post hoc Tukey test. Three independent replicates were obtained. (C) Inhibition of TGF $\beta$-induced fibrosis marker genes by prostaglandin receptor agonists in NHLFs. $N=3$, mean \pm S.D. $* P<0.05 ; * * P<0.005$; $* * * P<0.001$, compared with TGF $\beta$ but no compound treated group (orange bar). Two-way ANOVA post hoc Tukey test. Three independent replicates were obtained. RFU, relative fluorescence unit.

pleiotropic effect of PGE2 limits its applications in the broader patient population and in chronic usage. Side effects of PGE2 systemic administration include headache, uterine contraction, gastrointestinal disturbance, and diarrhea. As such, a substantial amount of effort has been put into developing subtype-selective prostanoid receptor agonists to obtain a better risk/benefit profile (Flesch et al., 2013). EP4 agonist ONO-4819CD has been tested in a phase 2 ulcerative colitis trial but failed to achieve a statistically significant therapeutic effect (Nakase et al., 2010). To date, no EP2, EP4, or EP2/4 dual agonist (antagonist) has been approved for clinical use. Developing a locally delivered PGE2 modulator, such as via inhalation and topical administration, may represent a viable approach for the treatment of fibrosis.

Our work has demonstrated that delineating a beneficial GPCR signaling pathway for fibrosis is valuable for identification of a new target. In our proof-of-concept study, we focused on an in vitro cell-based system. DREADDs chemogenetic tools have been previously used to manipulate neuronal activity and behavior in animals (Vardy et al., 2015). As such, it is conceivable that a similar approach can be used to probe GPCR signaling in multiple fibrosis animal models. Combined with transcriptome analysis of the GPCR family, it is possible to identify novel GPCR target(s) for fibrosis.

\section{Acknowledgments}

The authors thank Jennifer Cho for help with the RNA-seq and Gayathri Swaminath and Christopher Novotny for their critical reviews of the manuscript.

\section{Authorship Contributions}

Participated in research design: Zhang, Vardy, Pinto, Peier.

Conducted experiments: Zhang, Vardy, Vadlamudi.

Contributed new reagents or analytic tools: Visconti.

Performed data analysis: Muise, Wang.

Wrote or contributed to the writing of the manuscript: Zhang, Vardy, Muise, Wang, Visconti. 


\section{References}

Akhurst RJ and Hata A (2012) Targeting the TGF $\beta$ signalling pathway in disease. Nat Rev Drug Discov 11:790-811.

Ames RS, Kost TA, and Condreay JP (2007) BacMam technology and its application to drug discovery. Expert Opin Drug Discov 2:1669-1681.

Armbruster BN, Li X, Pausch MH, Herlitze S, and Roth BL (2007) Evolving the lock to fit the key to create a family of G protein-coupled receptors potently activated by an inert ligand. Proc Natl Acad Sci USA 104:5163-5168.

Armstrong MJ, Gaunt P, Aithal GP, Barton D, Hull D, Parker R, Hazlehurst JM, Guo K, Abouda G, Aldersley MA, et al.; LEAN trial team (2016) Liraglutide safety and efficacy in patients with non-alcoholic steatohepatitis (LEAN) a multicentre, double-blind, randomised, placebo-controlled phase 2 study. Lancet 387:679-690.

Billot X, Chateauneuf A, Chauret N, Denis D, Greig G, Mathieu MC, Metters KM, Slipetz DM, and Young RN (2003) Discovery of a potent and selective agonist of the prostaglandin EP4 receptor. Bioorg Med Chem Lett 13:1129-1132.

Bologa CG, Revankar CM, Young SM, Edwards BS, Arterburn JB, Kiselyov AS, Parker MA, Tkachenko SE, Savchuck NP, Sklar LA, et al. (2006) Virtual and biomolecular screening converge on a selective agonist for GPR30. Nat Chem Biol 2:207-212.

Bozyk PD and Moore BB (2011) Prostaglandin E2 and the pathogenesis of pulmonary fibrosis. Am J Respir Cell Mol Biol 45:445-452.

Calder PC (2009) Polyunsaturated fatty acids and inflammatory processes: new twists in an old tale. Biochimie 91:791-795.

Coleman RA, Humphrey PP, Kennedy I, Levy GP, and Lumley P (1981) Comparison of the actions of U-46619, a prostaglandin $\mathrm{H} 2$-analogue, with those of prostaglandin $\mathrm{H} 2$ and thromboxane $\mathrm{A} 2$ on some isolated smooth muscle preparations. $\mathrm{Br}$ $J$ Pharmacol 73:773-778.

DeNinno MP, Schoenleber R, MacKenzie R, Britton DR, Asin KE, Briggs C, Trugman JM, Ackerman M, Artman L, Bednarz L, et al. (1991) A68930: a potent agonist selective for the dopamine D1 receptor. Eur J Pharmacol 199:209-219.

Deschamps AM and Murphy E (2009) Activation of a novel estrogen receptor, GPER, is cardioprotective in male and female rats. Am J Physiol Heart Circ Physiol 297: H1806-H1813.

Duffield JS (2014) Cellular and molecular mechanisms in kidney fibrosis. J Clin Invest 124:2299-2306.

Elwakeel E, Brüne B, and Weigert A (2019) $\mathrm{PGE}_{2}$ in fibrosis and cancer: insights into fibroblast activation. Prostaglandins Other Lipid Mediat 143:106339.

Flesch D, Merk D, Lamers C, and Schubert-Zsilavecz M (2013) Novel prostaglandin receptor modulators--part II: EP receptor modulators; a patent review (2002 2012). Expert Opin Ther Pat 23:233-267.

Fribourg M, Moreno JL, Holloway T, Provasi D, Baki L, Mahajan R, Park G, Adney SK, Hatcher C, Eltit JM, et al. (2011) Decoding the signaling of a GPCR heteromeric complex reveals a unifying mechanism of action of antipsychotic drugs. Cell 147:1011-1023.

Friedman SL, Ratziu V, Harrison SA, Abdelmalek MF, Aithal GP, Caballeria J, Francque S, Farrell G, Kowdley KV, Craxi A, et al. (2018) A randomized, placebocontrolled trial of cenicriviroc for treatment of nonalcoholic steatohepatitis with fibrosis. Hepatology 67:1754-1767.

Friedman SL, Sheppard D, Duffield JS, and Violette S (2013) Therapy for fibrotic diseases: nearing the starting line. Sci Transl Med 5:167sr1.

Gardell LR, Ma JN, Seitzberg JG, Knapp AE, Schiffer HH, Tabatabaei A, Davis CN, Owens M, Clemons B, Wong KK, et al. (2008) Identification and characterization of novel small-molecule protease-activated receptor 2 agonists. J Pharmacol Exp Ther 327:799-808.

Gilman AG (1987) G proteins: transducers of receptor-generated signals. Annu Rev Biochem 56:615-649.

Gruber CW, Koehbach J, and Muttenthaler M (2012) Exploring bioactive peptides from natural sources for oxytocin and vasopressin drug discovery. Future Med Chem 4:1791-1798.

Guettier JM, Gautam D, Scarselli M, Ruiz de Azua I, Li JH, Rosemond E, Ma X Gonzalez FJ, Armbruster BN, Lu H, et al. (2009) A chemical-genetic approach to study G protein regulation of beta cell function in vivo. Proc Natl Acad Sci USA 106:19197-19202.

Harrison SA, Rinella ME, Abdelmalek MF, Trotter JF, Paredes AH, Arnold HL, Kugelmas M, Bashir MR, Jaros MJ, Ling L, et al. (2018) NGM282 for treatment of non-alcoholic steatohepatitis: a multicentre, randomised, double-blind, placebocontrolled, phase 2 trial. Lancet 391:1174-1185.

Hauser AS, Attwood MM, Rask-Andersen M, Schiöth HB, and Gloriam DE (2017) Trends in GPCR drug discovery: new agents, targets and indications. Nat Rev Drug Discov 16:829-842.

Jensen MS, Mutsaers HAM, Tingskov SJ, Christensen M, Madsen MG, Olinga P, Kwon TH, and Nørregaard R (2019) Activation of the prostaglandin $\mathrm{E}_{2} \mathrm{EP}_{2} \mathrm{re}$ ceptor attenuates renal fibrosis in unilateral ureteral obstructed mice and human kidney slices. Acta Physiol (Oxf) 227:e13291.

Jo E, Bhhatarai B, Repetto E, Guerrero M, Riley S, Brown SJ, Kohno Y, Roberts E, Schürer SC, and Rosen H (2012) Novel selective allosteric and bitopic ligands for the S1P(3) receptor. ACS Chem Biol 7:1975-1983

Kang L, McIntyre KW, Gillooly KM, Yang Y, Haycock J, Roberts S, Khanna A Herpin TF, Yu G, Wu X, et al. (2006) A selective small molecule agonist of the melanocortin-1 receptor inhibits lipopolysaccharide-induced cytokine accumulation and leukocyte infiltration in mice. J Leukoc Biol 80:897-904.

Kolodsick JE, Peters-Golden M, Larios J, Toews GB, Thannickal VJ, and Moore BB (2003) Prostaglandin E2 inhibits fibroblast to myofibroblast transition via E. prostanoid receptor 2 signaling and cyclic adenosine monophosphate elevation. Am $J$ Respir Cell Mol Biol 29:537-544.

Lama V, Moore BB, Christensen P, Toews GB, and Peters-Golden M (2002) Prostaglandin E2 synthesis and suppression of fibroblast proliferation by alveolar epithelial cells is cyclooxygenase-2-dependent. Am J Respir Cell Mol Biol 27:752-758.
Mallat A, Teixeira-Clerc F, and Lotersztajn S (2013) Cannabinoid signaling and liver therapeutics. $J$ Hepatol 59:891-896.

Markovič T, Jakopin Ž, Dolenc MS, and Mlinarič-Raščan I (2017) Structural features of subtype-selective EP receptor modulators. Drug Discov Today 22:57-71.

Nakase H, Fujiyama Y, Oshitani N, Oga T, Nonomura K, Matsuoka T, Esaki Y, Murayama T, Teramukai S, Chiba T, et al. (2010) Effect of EP4 agonist (ONO-4819CD) for patients with mild to moderate ulcerative colitis refractory to 5-aminosalicylates: a randomized phase II, placebo-controlled trial. Inflamm Bowel Dis 16:731-733.

Nanthakumar CB, Hatley RJ, Lemma S, Gauldie J, Marshall RP, and Macdonald SJ (2015) Dissecting fibrosis: therapeutic insights from the small-molecule toolbox. Nat Rev Drug Discov 14:693-720.

Nawaratne V, Leach K, Suratman N, Loiacono RE, Felder CC, Armbruster BN, Roth BL, Sexton PM, and Christopoulos A (2008) New insights into the function of M4 muscarinic acetylcholine receptors gained using a novel allosteric modulator and a DREADD (designer receptor exclusively activated by a designer drug). Mol Pharmacol 74:1119-1131.

Neuschwander-Tetri BA, Loomba R, Sanyal AJ, Lavine JE, Van Natta ML, Abdelmalek MF, Chalasani N, Dasarathy S, Diehl AM, Hameed B, et al.; NASH Clinical Research Network (2015) Farnesoid X nuclear receptor ligand obeticholic acid for non-cirrhotic, non-alcoholic steatohepatitis (FLINT): a multicentre, randomised, placebo-controlled trial. Lancet 385:956-965.

Palmer SM, Snyder L, Todd JL, Soule B, Christian R, Anstrom K, Luo Y, Gagnon R, and Rosen G (2018) Randomized, double-blind, placebo-controlled, phase 2 trial of BMS-986020, a lysophosphatidic acid receptor antagonist for the treatment of idiopathic pulmonary fibrosis. Chest 154:1061-1069.

Patsenker E and Stickel F (2016) Cannabinoids in liver diseases. Clin Liver Dis (Hoboken) 7:21-25.

Penke LR, Huang SK, White ES, and Peters-Golden M (2014) Prostaglandin E2 inhibits $\alpha$-smooth muscle actin transcription during myofibroblast differentiation via distinct mechanisms of modulation of serum response factor and myocardinrelated transcription factor-A. J Biol Chem 289:17151-17162.

Perez-García GS and Meneses A (2005) Effects of the potential 5-HT7 receptor agonist AS 19 in an autoshaping learning task. Behav Brain Res 163: 136-140

Porter RH, Benwell KR, Lamb H, Malcolm CS, Allen NH, Revell DF, Adams DR, and Sheardown MJ (1999) Functional characterization of agonists at recombinant human 5-HT2A, 5-HT2B and 5-HT2C receptors in CHO-K1 cells. Br J Pharmacol 128:13-20.

Premont RT and Gainetdinov RR (2007) Physiological roles of G protein-coupled receptor kinases and arrestins. Annu Rev Physiol 69:511-534.

Ratziu V, Harrison SA, Francque S, Bedossa P, Lehert P, Serfaty L, Romero-Gomez M, Boursier J, Abdelmalek M, Caldwell S, et al.; GOLDEN-505 Investigator Study Group (2016) Elafibranor, an agonist of the peroxisome proliferator-activated receptor-alpha and -delta, induces resolution of nonalcoholic steatohepatitis without fibrosis worsening. Gastroenterology 150:1147-1159.e5.

Revankar CM, Cimino DF, Sklar LA, Arterburn JB, and Prossnitz ER (2005) A transmembrane intracellular estrogen receptor mediates rapid cell signaling. Science 307:1625-1630

Ring RH, Schechter LE, Leonard SK, Dwyer JM, Platt BJ, Graf R, Grauer S, Pulicicchio C, Resnick L, Rahman Z, et al. (2010) Receptor and behavioral pharmacology of WAY-267464, a non-peptide oxytocin receptor agonist. Neuropharmacology 58:69-77.

Sam AH, Salem V, and Ghatei MA (2011) Rimonabant: from RIO to ban. J Obes 2011 432607.

Schanstra JP, Neau E, Drogoz P, Arevalo Gomez MA, Lopez Novoa JM, Calise D, Pecher C, Bader M, Girolami JP, and Bascands JL (2002) In vivo bradykinin B2 receptor activation reduces renal fibrosis. J Clin Invest 110:371-379.

Schiller M, Dennler S, Anderegg U, Kokot A, Simon JC, Luger TA, Mauviel A and Böhm M (2010) Increased cAMP levels modulate transforming growth factorbeta/Smad-induced expression of extracellular matrix components and other key fibroblast effector functions. J Biol Chem 285:409-421.

Schlessinger K, Li W, Tan Y, Liu F, Souza SC, Tozzo E, Liu K, Thompson JR, Wang L, and Muise ES (2015) Gene expression in WAT from healthy humans and monkeys correlates with FGF21-induced browning of WAT in mice. Obesity (Silver Spring) 23:1818-1829.

Sieber P, Schäfer A, Lieberherr R, Le Goff F, Stritt M, Welford RWD, Gatfield J, Peter O, Nayler O, and Lüthi U (2018) Novel high-throughput myofibroblast assays identify agonists with therapeutic potential in pulmonary fibrosis that act via EP2 and EP4 receptors. PLoS One 13:e0207872.

Sumner MJ, Cannon TR, Mundin JW, White DG, and Watts IS (1992) Endothelin ETA and ETB receptors mediate vascular smooth muscle contraction. $\mathrm{Br} J$ Pharmacol 107:858-860.

Tager AM, LaCamera P, Shea BS, Campanella GS, Selman M, Zhao Z, Polosukhin V, Wain J, Karimi-Shah BA, Kim ND, et al. (2008) The lysophosphatidic acid receptor LPA1 links pulmonary fibrosis to lung injury by mediating fibroblast recruitment and vascular leak. Nat Med 14:45-54.

Thomas PE, Peters-Golden M, White ES, Thannickal VJ, and Moore BB (2007) PGE(2) inhibition of TGF-beta1-induced myofibroblast differentiation is Smadindependent but involves cell shape and adhesion-dependent signaling. Am J Physiol Lung Cell Mol Physiol 293:L417-L428.

Tintner R, Manian P, Gauthier P, and Jankovic J (2005) Pleuropulmonary fibrosis after long-term treatment with the dopamine agonist pergolide for Parkinson Disease. Arch Neurol 62:1290-1295.

Urban DJ and Roth BL (2015) DREADDs (designer receptors exclusively activated by designer drugs): chemogenetic tools with therapeutic utility. Annu Rev Pharmacol Toxicol 55:399-417.

Vardy E, Robinson JE, Li C, Olsen RHJ, DiBerto JF, Giguere PM, Sassano FM, Huang XP, Zhu H, Urban DJ, et al. (2015) A new DREADD facilitates the multiplexed chemogenetic interrogation of behavior. Neuron 86:936-946. 
Weis WI and Kobilka BK (2018) The molecular basis of G protein-coupled receptor activation. Annu Rev Biochem 87:897-919.

Wettlaufer SH, Scott JP, McEachin RC, Peters-Golden M, and Huang SK (2016) Reversal of the transcriptome by prostaglandin E2 during myofibroblast dedifferentiation. Am J Respir Cell Mol Biol 54:114-127.

Wiernas TK, Griffin BW, and Sharif NA (1997) The expression of functionallycoupled B2-bradykinin receptors in human corneal epithelial cells and their pharmacological characterization with agonists and antagonists. $\mathrm{Br} J$ Pharmacol 121:649-656.

Wilborn J, Crofford LJ, Burdick MD, Kunkel SL, Strieter RM, and Peters-Golden M (1995) Cultured lung fibroblasts isolated from patients with idiopathic pulmonary fibrosis have a diminished capacity to synthesize prostaglandin E2 and to express cyclooxygenase-2. J Clin Invest 95:1861-1868.
Wynn TA and Ramalingam TR (2012) Mechanisms of fibrosis: therapeutic translation for fibrotic disease. Nat Med 18:1028-1040.

Zhang J, Muise ES, Han S, Kutchukian PS, Costet P, Zhu Y, Kan Y, Zhou H, Shah V, Huang H, et al. (2020) Molecular profiling reveals a common metabolic signature of tissue fibrosis. Cell Rep Med 1:1-11.

Address correspondence to: Andrea M. Peier, Departments of Screening and Compound Profiling, MRL, Merck \& Co., Inc., 2000 Galloping Hill Rd., Kenilworth, NJ 07033. E-mail: andrea_peier@merck.com; or Ji Zhang, Departments of Cardiometabolic Diseases, MRL, Merck \& Co., Inc., 2000 Galloping Hill Rd., Kenilworth, NJ 07033. E-mail: jzhang30@gmail.com 